\title{
Drip Control Automation System for Hospitals
}

\author{
Mr. Manoj Vishnoi, Deepak Gupta, Abhishek Singh, Avni \\ Department of Electronics and Communication Engineering SRM University, NCR Campus, Ghaziabad
}

\begin{abstract}
The main aim of this project is to relieve human effort by automatically controlling the dripping system of the patient in a hospital. This project consists of a RF transmitter and receiver which is basically used for the wireless communication purpose. In this an alert message will be sent to the concern doctor even can control the drip system as the drip reaches a particular level by using GSM module. The PIC16F876A microcontroller is used to processes the information for all the instructions given to each component. A load cell is used to measure the weight of the drip interfaced with IC hx711 that provide the level information to microcontroller. An external actuator is used just next to the drip which is an electromagnetic valve that blocks the flow of liquid to the patient body as the drip reaches the particular level and also starts the drip system by receiving of the message from the concerned doctor.
\end{abstract}

\section{Introduction}

With the help of this technology we can check the level of fluid given to the patients automatically without doing any manual observation. We need to set the level of the dripping bottle at some point and when it reaches just below the set level it sends an alert message to the phone of the concerned doctor along with a buzzer. After which it depends on the doctor weather to stop the drip at that point itself or the drip must continue, and this can be just done by sending a message by using GSM module. The data is transferred from the transmitter to the receiver through radio frequency, for which an RF module is used. The RF module Consist of a RF transmitter and a RF receiver [1]. RF transmitter performs modulation, up-conversion, and power amplification, with the first two function combined in some cases. The design of RF transmitters for wireless application entails many challenges at both architecture and circuit level. There is an RF interface in the transmitter which performs the desired modulation and up-conversion.

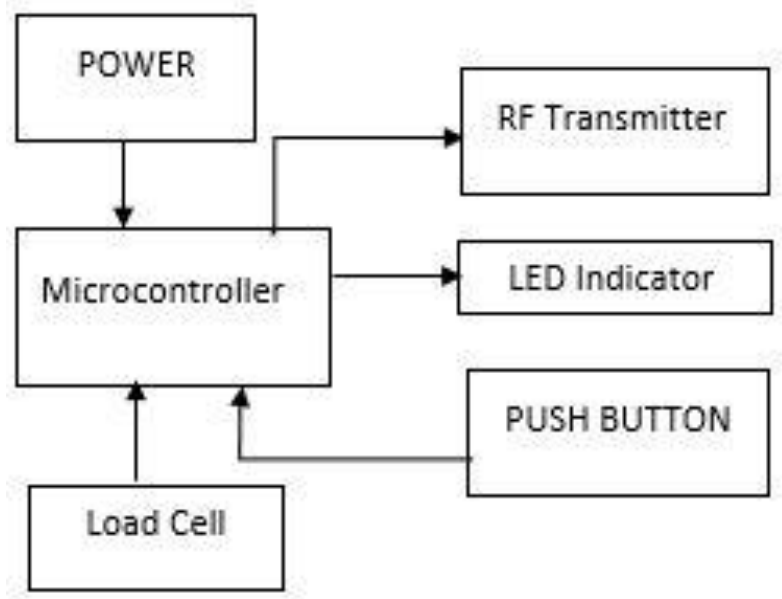

Figure 1 RF Transmitter side BLOCK diagram

The RF module operates at a frequency of $434 \mathrm{MHz}$. An RF Transmitter receives serial data and transmits it wirelessly through its antenna connected at pin 4 . The transmission occurs at the rate of $1 \mathrm{Kbps}-$ $10 \mathrm{Kbps}$. The transmitted data is received by an RF receiver operating at the same frequency as that of the transmitter.

Load cell is connected to the microcontroller. Among the complete family of sensors and transducers for automotive, research, consumers and industrial applications, load cells stand out as one of the most important transducers [2]. A hook is attached to the load cell to hang the dripping bottle where it measures the mechanical weight of the dripped fluid and convert it to the electrical weight, and sends the measured data to the microcontroller. 


\begin{tabular}{|c|l|l|}
\hline Pin No & Function & Name \\
\hline 1 & Ground (OV) & Ground \\
\hline 2 & Serial data input pin & Data \\
\hline 3 & Supply voltage; 5V & VCC \\
\hline 4 & Antenna output pin & ANT \\
\hline
\end{tabular}

Table 1- Pin Configuration of RF transmitter

An RF receiver module receives the modulated RF signal, and demodulates it. At receiver end we have similar connection for power supply as microcontroller needs +5 volts. The pin diagram of the RF receiver is mentioned in table-2.

\begin{tabular}{|c|l|l|}
\hline Pin No & Function & Name \\
\hline 1 & Ground (OV) & Ground \\
\hline 2 & Serial data output pin & Data \\
\hline 3 & Linear output pin; not connected & NC \\
\hline 4 & Supply voltage; $5 \mathrm{~V}$ & Vcc \\
\hline 5 & Supply voltage; $5 \mathrm{~V}$ & Vcc \\
\hline 6 & Ground (0V) & Ground \\
\hline 7 & Ground (OV) & Ground \\
\hline 8 & Antenna input pin & ANT \\
\hline
\end{tabular}

Table 2- Pin configuration of RF Receiver

The receiver side of the project consist of the GSM module, LCD, Microcontroller, actuator and RF receiver module. The block diagram of the receiver side is given in figure 4 .

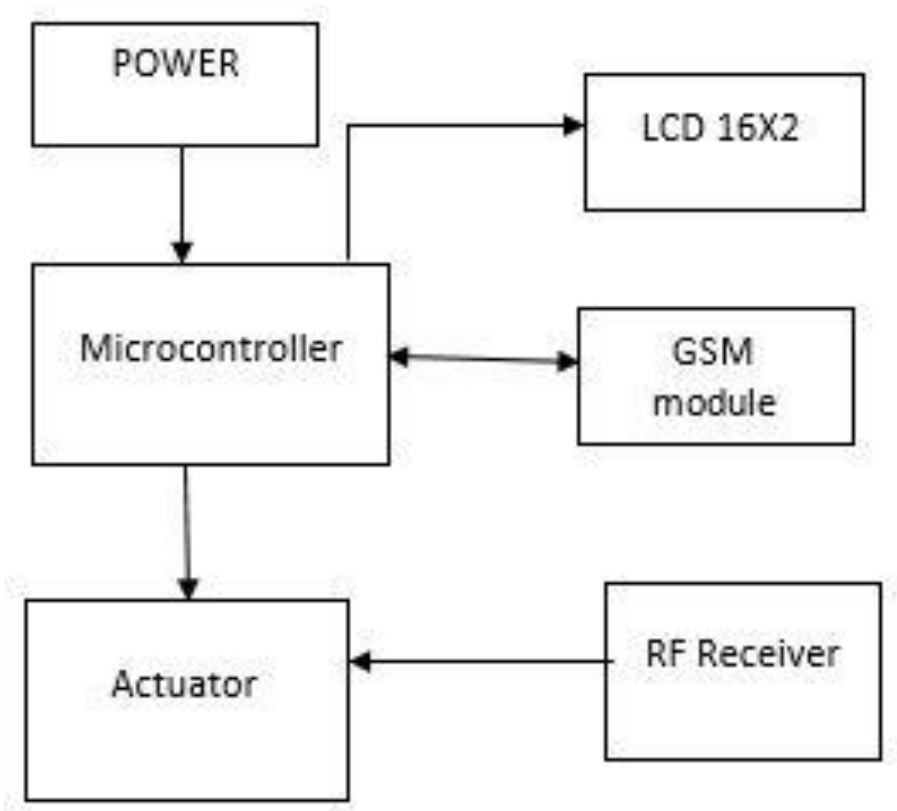

Figure 2- Block Diagram Receiver side 


\section{Construction}

In this there are two parts i.e., the transmitter part and second the receiver part. Both the transmitter and the receiver are connected through radio frequency. In the transmitter part a load cell is connected which is used to measure the weight of the fluid dripped. In the transmitter part there is a voltage regulator connected to the power supply. The voltage regulator provides 5 volt power supply to the controller. The transmitter part also consists of RF transmitter module. It transmits the data to the receiver RF module. The microcontroller used is PIC16F876A.

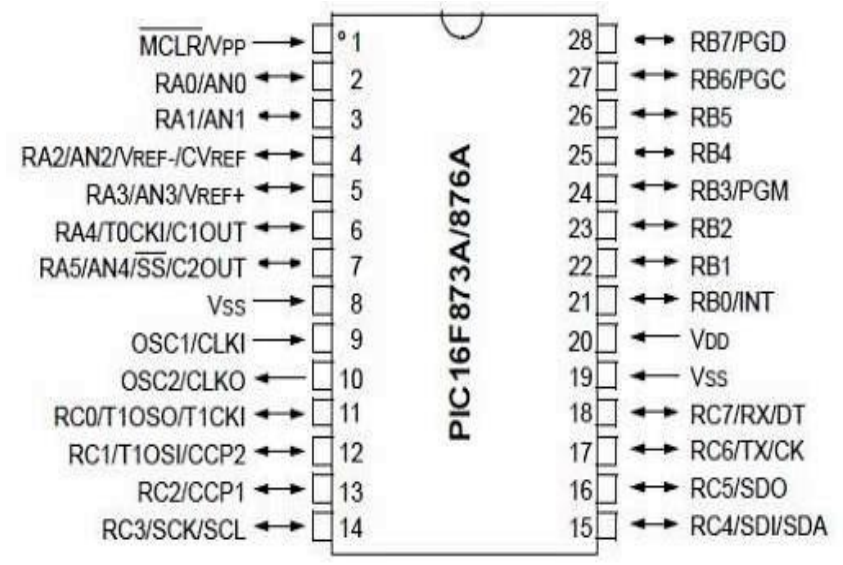

Figure 3- PIC16F876A pin diagram

The pin diagram of PIC16F876A is shown in figure 3.

The circuit diagram for transmitter side is shown in fig. 4 The RF module is connected to the pic microcontroller. There are 4 indicator LEDs connected to the microcontroller to the pins RB1, RB2, RB3 and RB4.

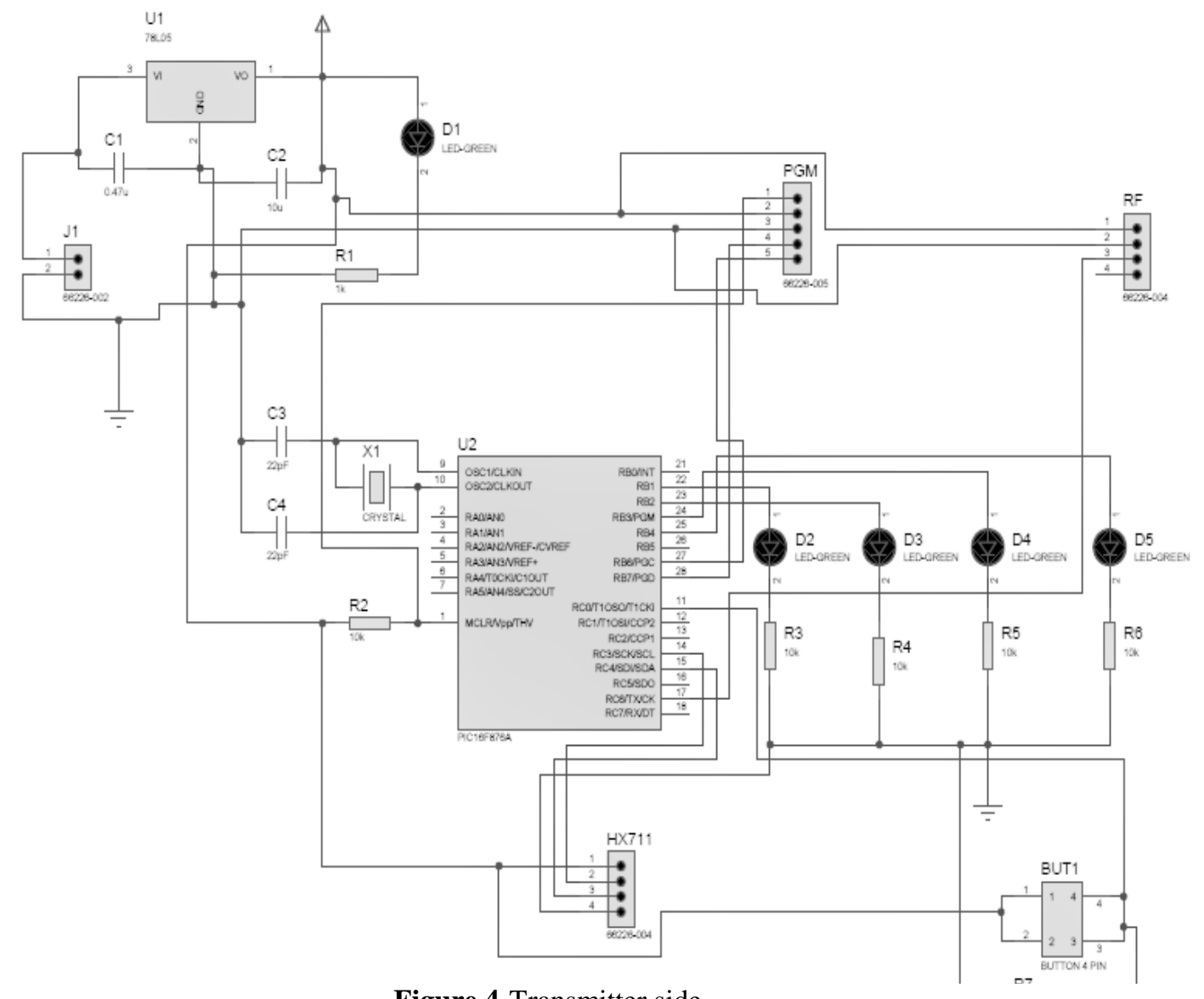

Figure 4-Transmitter side 
Pin Tx of microcontroller is connected to the RF transmitter. Pin RC3 and RC 4 are connected to pin 2 and pin 3 of HX711 respectively. Hx711 is a load cell amplifier which is used to get miserable data out from a load cell. In the receiver part The GSM module is connected to the pin Tx of PIC16F876A. The circuit diagram for the receiver side is shown in figure 5 .

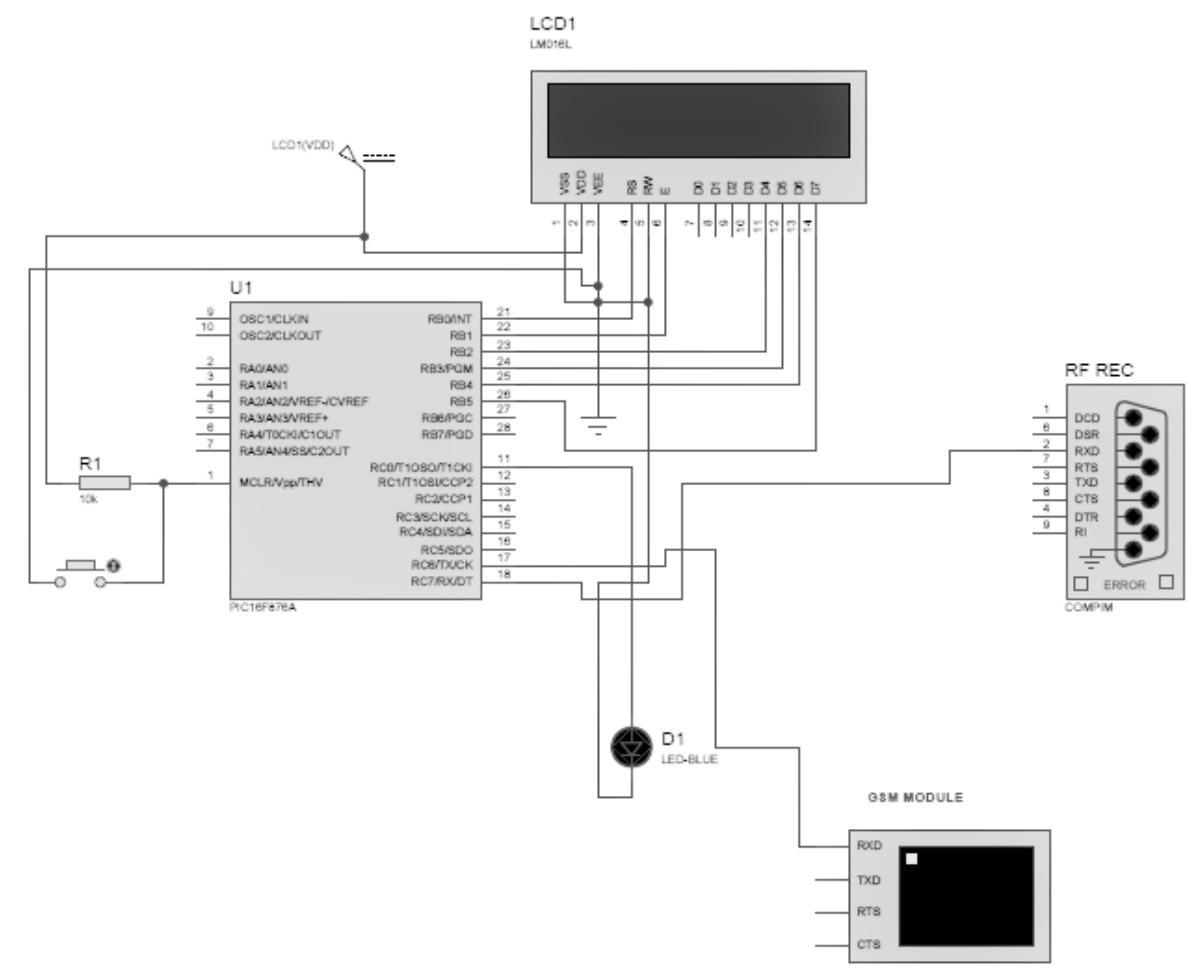

Figure 5- Receiver Side

Pin 2 of $\mathrm{RF}$ receiver is connected to pin $\mathrm{Rx} / \mathrm{RC} 7$ of pic16f876a microcontroller. LCD is also connected to the microcontroller as per the pin diagram shown in the figure 6.

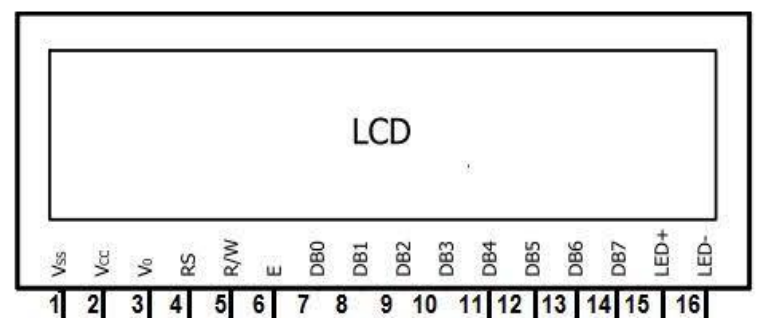

Figure 6- LCD pin diagram

\section{Working}

In this prototype we connect a voltage regulator to the microcontroller which is used to supply a constant voltage of 5volts. The transmitter part consist of a load cell. Load cell is a transducer that is used to create an electrical signal whose magnitude proportional to the force being measured. The load cell here is used to measure the weight of the fluid to be dripped which is hanged through a hook connected with the load cell. There are 3 LEDs connected for the level indication of the fluid for 3 different levels. An RF module is used both at the transmitter and the receiver side. RF module is a small electronic device used to transmit and/or receive radio signals between two devices. This wireless communication can be accomplished through Radio Frequency (RF) communication. The level can be set through a trigger. The weight of the dripped fluid is continuously monitored and the RF transmitter transmits the data to the RF receiver. When the weight of the fluid present inside the bottle reaches below the set level the buzzer gets started and a message is send to the concerned doctor through GSM module. An electrically operated valve is connected to the bottle. When the fluid reaches the set level the valve gets closed and the dripping gets stopped. If the concerned doctor wants to start the dripping then he/she can send a message and the valve gets opened until the dripping reaches the next level. 


\section{Conclusion}

The weight of the dripped fluid is measured through the load cell. The RF module is used for the communication between the transmitter and the receiver over radio frequency. The GSM module is used to send or receive message to or from the concerned doctor to stop the drip or to get the drip started. This prototype helps proper monitoring of the fluid even in the absence of any doctor or nurse thereby assuring complete safety of the patients' health.

\section{References}

[1] BehzadRazavi, "RF transmitter Architecture and circuit" from University of California. IEEE 0-7803-5443-5/99.

[2] Wilmar Hernandez, "Improving the response of a load cell by using optimal filtering", Sensors 2006, July.

[3] Priyadharshani.R, Mithuna.S, VasanthKumar.U, KalpanaDevi.S, Dr. SuthanthiraVanitha.N, "Automatic Intravenous Fluid Level Indication System for Hospitals", Volume 3 Issue VIII, august 2015 ISSN: 2321-9653.

[4] Microchip Technology PIC16F87XA Data Sheet 28/40/44-Pin Enhanced Flash Microcontrollers. 